\title{
On the factorization of composite polymer knots into separated prime components
}

\author{
Luca Tubiana \\ Department of Theoretical Physics, Jožef Stefan Institute, \\ Jamova cesta 39, SI-1000 Ljubljana (Slovenia)* and \\ SISSA - Scuola Internazionale Superiore di Studi Avanzati Via Bonomea 265, 34136 Trieste (Italy)
}

(Dated: August 22, 2018)

\begin{abstract}
Using Monte Carlo simulations and advanced knot localization methods, we analyze the length and distribution of prime components in composite knots tied on Freely Jointed Rings (FJRs). For increasing contour length, we observe the progressive factorization of composite knots into separated prime components. However, we observe that a complete factorization, equivalent to the "decorated ring" picture, is not obtained even for rings of contour lengths $N \simeq 3 N_{0}$, about tens of times the most probable length of the prime knots tied on the rings. The decorated ring hypothesis has been used in literature to justify the factorization of composite knots probabilities into the knotting probabilities of their prime components. Following our results, we suggest that such hypothesis may not be necessary to explain the factorization of the knotting probabilities, at least when polymers excluded volume is not relevant. We rationalize the behavior of the system through a simple one dimensional model in which prime knots are replaced by sliplinks randomly placed on a circle, with the only constraint that the length of the loops has the same distribution of the length of the corresponding prime knots.
\end{abstract}

\section{INTRODUCTION}

Knots are known to affect the physical properties of polymers [1-7], as well as the functionality of biopolymers such as DNA $[8,9]$. Knots have been found and are actively studied in proteins [10-15], and in viral genomic DNA [16, 17] where they can be used as a fingerprint to study genome packaging in bacteriophages [18, 19]. Furthermore, several recent studies brought under the light the relevance of knots in nanotechnological applications [7, 20-23].

Physical knots appear and diffuse spontaneously on polymer chains [24-27] and can be trapped by cyclization of the chains' ends. It is possible, and frequent, for several knots to appear on the same chain, which is then said to host a composite knot (see Fig. 1 e)); in fact, it has been mathematically proved that such configurations are by far the most probable for long polymers [24, 28]. Nonetheless, the majority of studies on the physical properties of knotted polymers still focus on simple (prime) knots, while composite knots have been studied mostly in particular systems, like quasi-2d rings [29, 30], strong polyelectrolites [31] or ring confined into nano-channels [21]. In all those systems, numerical studies shown that if several prime knots are tied on the same chain, they do not intermingle and behave independently one from another; composite knots are therefore "factorized" into independent localized prime components and can be thought as decorated rings, like the one depicted in Fig. $1 \mathrm{f}$ ). This analogy, supported by the observed weak localization of prime knots [32-34], is invoked as well to explain the factorization of the knotting probability of very long three dimensional composite knotted rings into the product of the knotting probabilities of the prime knots involved [35-37]. However, to the best of our knowledge, no systematic study exists which reports on the onset of knot factorization with increasing ring contour length; aside for the particular systems discussed above, the validity of the assumption of complete knot factorization has not been tested. In this study we intend to fulfill this gap for Freely Jointed Rings, equilateral polygon with infinitely thin edges, presenting a detailed analysis of the relationship between ring contour length and knot factorization.

Using advanced knot localization algorithms [38], we are able to assign a knot length to composite knots, to identify all prime components which are separated along the ring, i.e. are not included into or intermingled with other prime components, to measure their length, the distribution of distances between nearest neighbors separated prime components and the probability of having a complete factorization of composite knots into separated prime components.

We show that even for rings whose contour length is about three times the unknotting length, $N_{0}$, that is tens of times the most probable length of the prime knots considered, the probability of still having an overlap of two or more prime components is not negligible, going from $\simeq 10 \%$ for the simplest composite knot, $3_{1} 3_{1}$, to $\simeq 45 \%$ for the most complex composite knot considered in this study, $3_{1} 3_{1} 3_{1} 4_{1}$. Nonetheless, for contour lengths $N \gtrsim N_{0}$, the length of

\footnotetext{
* luca.tubiana@ijs.si
} 


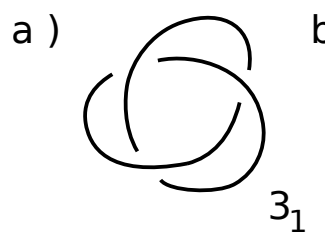

e )

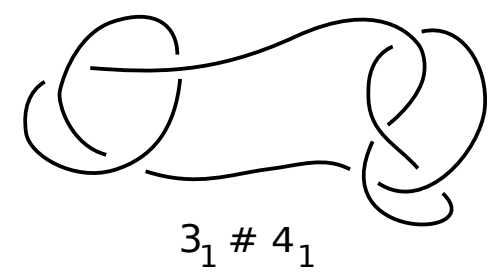

C )

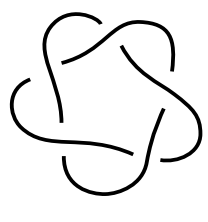

$5_{1}$

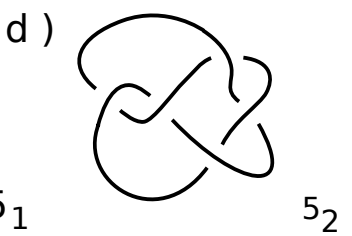

f )

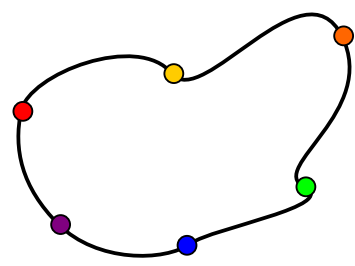

FIG. 1. The prime knot components considered in this work. a) $3_{1}$, b) $4_{1}$, c) $5_{1}$, d) $5_{2}$. Panel e) shows a simple composite knot with two prime components, $3_{1} 4_{1}$. f) In the limit of infinite length all component are expected to localize and behave like independent point like decorations along the ring [36, 37].

the composite knots as well as the distribution of distances between nearest neighbors separated prime components along the ring are compatible with a random placement of prime knots on the ring.

Our results can be rationalized through a simple one-dimensional model in which prime knots are substituted by loops enforced through sliplinks. Sliplinks can be imagined as buckle belt like rings which can move freely on the chain and enforce two distant part of it to come near each other [39]. As in ref. [39] we assume that loops can be nested within each other, but cannot be concatenated; we further assume that the loops length distributions coincide with the length distributions of the prime knots involved in the composite knot. We find that this simple model, with no free parameters, is able to reproduce qualitatively the knot factorization probability curves for increasing ring contour length, while it quantitatively reproduces the distribution of distances between separated nearest neighbors knots and the average composite knot lengths.

The article is organized as follows. Section II reports the simulation and analysis methods used throughout the study as well as the one-dimensional model. In section III we report the results obtained from the simulations, which are then compared with our one-dimensional model and discussed in relation with previous works in section IV.

\section{MODEL AND METHODS}

\section{A. The model}

Using the crankshaft rotation algorithm [40], we generated freely jointed equilateral rings of length $N=100,200, \ldots, 1000$; for comparison, the unknotting length of FJRs is $N_{0} \simeq 300$ [41]. The range of lengths considered spans the crossover from prime knots domination of the knot spectrum to composite knots domination; composite knots start to dominate the knot spectrum for $N \gtrsim 400$, in particular 2-components knots start from $N \simeq 550$ and 3-components knots from $N \simeq 900$, as reported in Fig. 2 .

For every length we generated $\simeq 10^{7}$ independent configurations with unconstrained topology. Rings with the desired topologies were extracted from the pool of freely jointed equilateral rings using the KNOTFIND algorithm included in the KnotScape package [42]. We considered knots composed by 2, 3 and 4 prime components, where all but one prime components were $3_{1}$ knots and the remaining one was either a $3_{1}, 4_{1}, 5_{1}$ or $5_{2}$ knot (e.g. $3_{1} 3_{1}, 3_{1} 4_{1}$, ..., $\left.3_{1} 3_{1} 5_{1}, 3_{1} 3_{1} 5_{2}, \ldots\right) .3_{1} 3_{1} 3_{1} 3_{1}$ and $3_{1} 3_{1} 3_{1} 4_{1}$ were the only knots with 4 components considered in this study. For every topology and every value of $N$ we collected several hundreds to thousands independent configurations.

\section{B. Analysis}

Consider a rope with several knots tied on it. We can intuitively say that one of them is separated from the others if we can cut it away and rejoin the rope without modifying any other knot tied on it. In the pictorial examples of Fig. 3, we can therefore find two separated components in panel b), one in c) and none in d). From a mathematical point of view though, this intuitive procedure is much subtler, as knots are defined only on closed rings; to assign a 


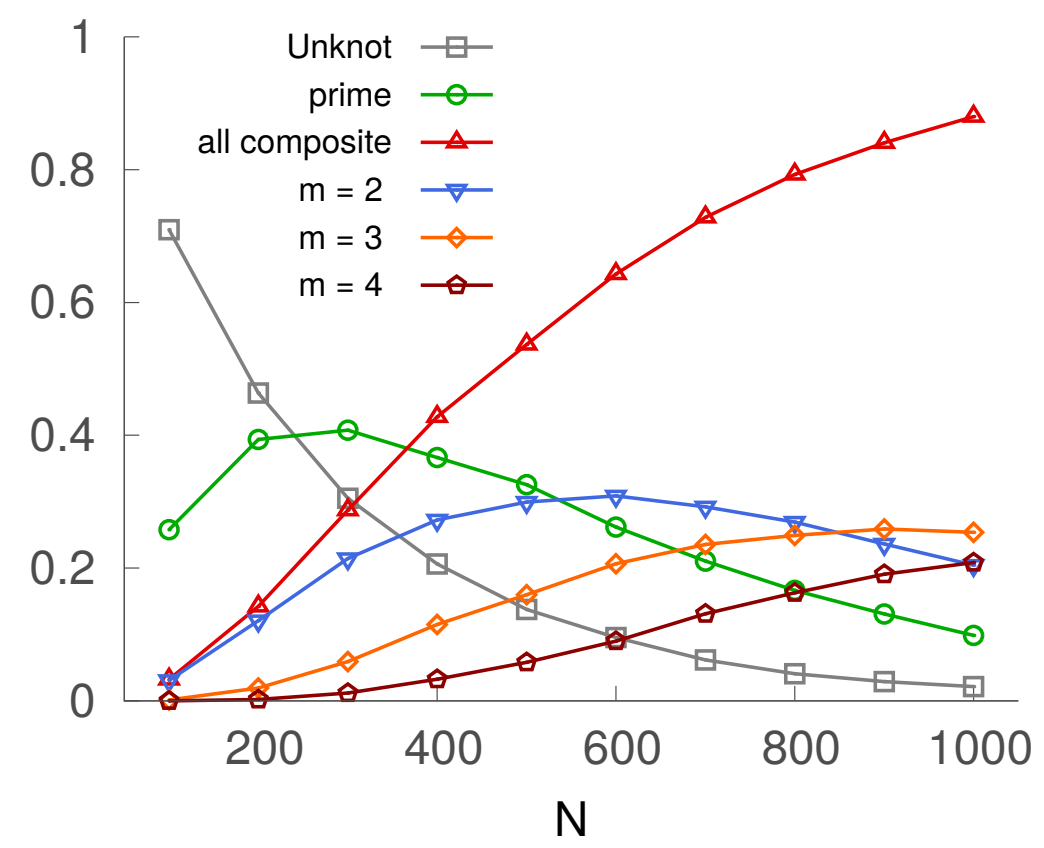

FIG. 2. Knot spectrum for Freely Jointed Rings of increasing contour length $N$, including unknot, prime and composite knots probabilities. For composite knots the plot reports the probability of a composite knots with an unrestricted number of components as well as those of composite knots with $m=2,3$ or 4 prime components. Note that composite knots become dominant for $N \gtrsim 400$, composite knots with $m=2$ components at $N \simeq 600$ and composite knots with $m=3$ components at $N \simeq 900$.

topological state to a given ring portion, which is an open arc, one must first close it into a ring, on which topological invariants can be evaluated. $[26,38,43,44]$ To close ring arcs and assign them a topological state, we adopt the minimally-interfering closure, a recently introduce closure scheme which is designed to introduce the least possible entanglement [38].

We consider a prime component of topology $\tau_{i}$ of a composite knot of topology $\tau_{\text {comp }}=\tau_{1} \ldots \tau_{i-1} \tau_{i} \tau_{i+1} \ldots \tau_{n}$ to be separated if we can identify, inside the composite-knotted portion of the ring, a subarc having topology $\tau_{i}$ whose complementary arc on the ring has topology $\tau_{\text {comp }} \backslash \tau_{i}=\tau_{1} \ldots \tau_{i-1} \tau_{i+1} \ldots \tau_{n}$. Fig 3 a), d) show a pictorial example of two prime components which are entangled to the point that it is impossible to find any arc satisfying the above conditions. The further condition that the prime knotted arcs must lie inside the composite-knotted portion of the ring, i.e. inside the shortest ring portion accommodating the whole composite knot, is set to guarantee consistency between all measured quantities.

To identify all separate prime components, we first identified the shortest knotted portion of the chain using a bottom-up search scheme [38], which identifies the shortest arc of the chain which has the desired topology along with an unknotted complementary arc. Then we ordered all the subarcs of the shortest knotted portion for increasing length. Starting from the shortest arc we checked if it was a factor prime knot of the composite knot. If this was the case, all the arcs overlapping it were removed from the list of arcs to be analyzed. This procedure guarantees that in a situation like that depicted in Fig. 3 c) only one component is seen as separated, while the larger knotted arc is discarded as it includes the smaller one and therefore can not be removed from the ring without removing the other knot along with it.

With the above procedure we computed the number of separated prime components in each configuration; the probability of having all prime components separated on a ring of length $N, P_{\text {sep }}\left(\tau_{c o m p}, N\right)$, estimated as the ratio between the number of configurations of topology $\tau_{\text {comp }}$ in which all prime components have been identified and the total number of configurations of that topology; the average length of separated prime components knots, $\left\langle l_{k}\right\rangle$, and the length of the composite knot, $\left\langle l_{k}^{\text {comp }}\right\rangle$.

\section{Ring simplification}

In order to reduce both the computational cost of localizing the knots and the incidence of slipknots [38, 45] we applied a topology-preserving simplification procedure. 


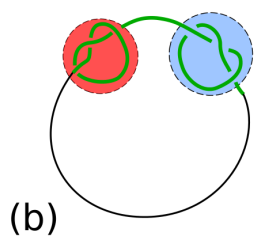

(b)

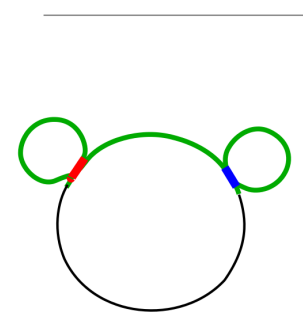

(e)

(h)

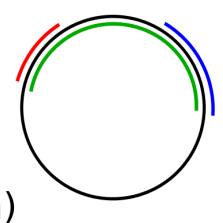

(a)

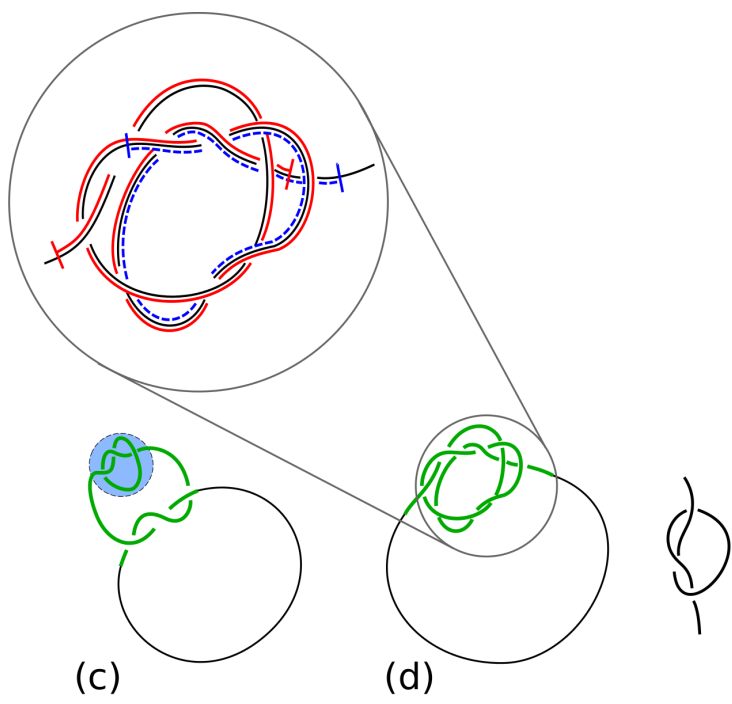

(f)
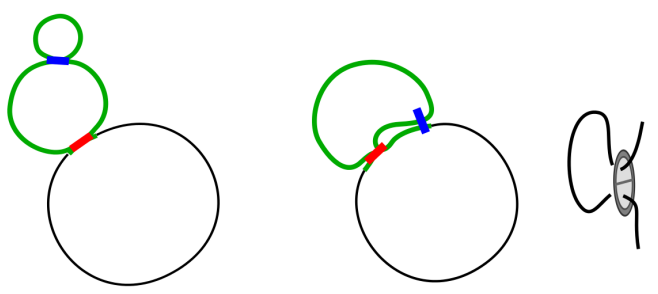

(g)

(i)

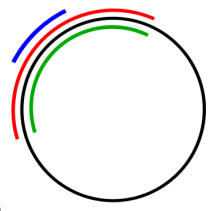

(j)

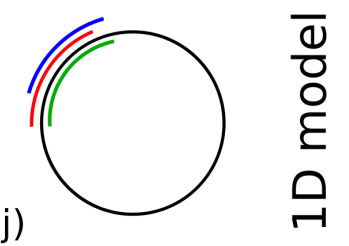

FIG. 3. a) Two prime components of a $3_{1} 3_{1}$ knot entangled in a way which does not allow the identification of a $3_{1}$ arc having complementary arc of topology $3_{1}$. The two arcs with topology $3_{1}$ are coloured in red and blue, but the condition on the complementary arc can not be satisfied for any of them. b), c) and d) $3_{1} 3_{1}$ knot with 2,1 and no separated prime components respectively. e), f), g): sliplink representations of knot configurations in panels b), c), d).

$\mathrm{h}$ ), i) and j): arc representations of the configurations in b), c) and d), used to implement sliplink configurations in the onedimensional model. Configurations like g) and j) are forbidden in our one-dimensional model. In panels b) to j) the knotted portion is highlighted in green while prime components are highlighted in red or blue.

The adopted simplification procedure is an improved version of the rectification scheme adopted in [10, 35, 46]. The standard rectification scheme consists in picking a random vertex $i$ of the ring and verify if it can be made collinear with its neighbor vertices $i-1$ and $i+1$ without crossing any edges of the ring. If it is possible, the vertex is eliminated. The procedure is then carried on until no more vertices can be eliminated. This simplification scheme alters the geometric properties of the ring, and can therefore modify some geometrical properties of the knots. In order to reduce these effects, we imposed a simplification stride, $s$, that limited the number of subsequent vertices that could be eliminated. We started with $s=2$ and applied the rectification scheme $N$ times, rejecting those vertex removals which would introduce a gap larger than $s$ between the remaining beads. We then repeated this procedure with a new value of $s, s^{\prime}=2 * s$ until we reached a maximum value of stride $s_{\max }=15$ which was chosen for it being close to the characteristic length of trefoil knots on FJRs (see supplementary information).

To obtain an estimation of the error introduced by the simplification procedure, we repeated the analysis on small sets of hundreds of configurations for several topologies for two other values of $s_{\max }: s_{\max }=4$ and $s_{\max }=\infty$. We observed that varying $s_{\max }$ the length of the composite knots remained compatible within the errors, while $P_{\text {sep }}$ diminished systematically for increasing $s_{\max }$, with a maximum difference in the estimate of $P_{\text {sep }}\left(3_{1} 3_{1} 3_{1} 3_{1}\right)$ of $\simeq 10 \%$ measured between $s_{\max }=4$ and $s_{\max }=\infty$. See supplementary information for further details. 


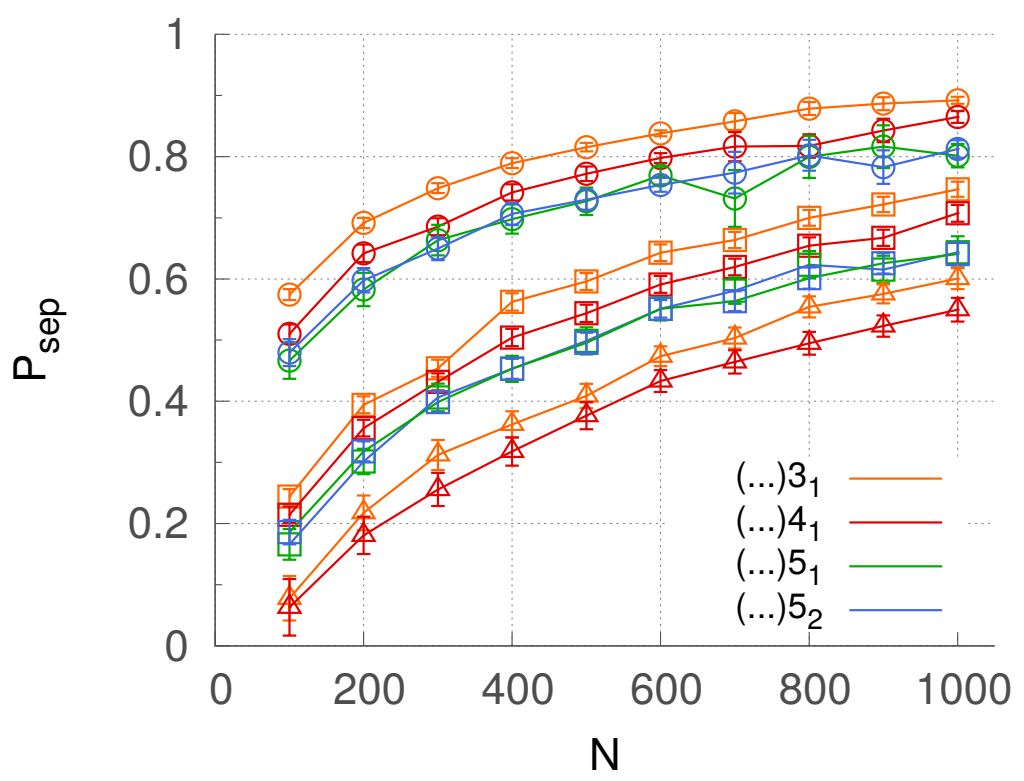

FIG. 4. Probability, $P_{\text {seq }}(\tau, N)$ of having all prime components separated, plotted as a function of ring length $N$, for different composite knots. From top to bottom, the lines report the probabilities for knots with 2,3 and 4 components with topologies $(\ldots) 3_{1},(\ldots) 4_{1},(\ldots) 5_{1},(\ldots) 5_{2}$ respectively.

\section{One-dimensional model}

To gain further insight into the behavior of composite knots, we designed a simple one-dimensional model in which prime factor knots are substituted with loops enforced by sliplinks [39] placed randomly on a plane, equilateral, polygon with $N$ edges, as illustrated in Fig. 3 e)-j). Any two loops on the polygon can only be either separated or nested within each other, so configurations like that of Fig. $3 \mathrm{~g}$ ) and j) are prohibited. The length distribution of the loops enforced by the sliplinks is assumed to be that of prime knots of the same topology. Following ref. [39] we call these structures formed by sliplinks on a ring "paraknots".

As $3_{1}$ and $4_{1}$ prime knots are those for which we have more statistics, we considered the following topologies: $3_{1} 3_{1}$, $3_{1} 4_{1}, 3_{1} 3_{1} 3_{1}, 3_{1} 3_{1} 4_{1}, 3_{1} 3_{1} 3_{1} 3_{1}$ and $3_{1} 3_{1} 3_{1} 4_{1}$. The length distribution of the loops is given by the empirical knot length distributions, $P\left(l_{k}, N\right)$, of prime knots $3_{1}$ and $4_{1}$ extracted from the pool of FJRs, evaluated with a bottom-up scheme (See Supplementary material). We stress that these are knot length distributions of prime knots, and not of prime components in a composite knot.

To generate paraknots with $m$ loops on polygons with $N$ edges, we first picked randomly $m$ edges of the polygon, corresponding to the starting points of the arcs representing the loops (in a clockwise direction). To every starting point we then associated a knot length picked randomly from the length distribution $P\left(l_{k}, N\right)$ of the corresponding prime knot $\left(3_{1}\right.$ or $\left.4_{1}\right)$ and computed the ending points of the arcs, taking into account the periodicity of the polygon. All configurations in which two or more arcs overlapped without one being strictly included into the other were rejected (e.g. Fig. 3 j)).

For each topology and ring length we generated several thousands paraknots configurations. On these configurations we performed the same analysis as on the configurations from the simulation. In this model, we defined the knotted portion of the "composite knot" as the shortest arc of the polygon which includes all the sliplinks, i.e. all the starting and ending points. Since a polygon with $m$ sliplinks is divided in $2 m$ "unknotted" arcs, the "knotted portion" is the complementary of the longest unknotted arc.

\section{RESULTS}

\section{A. Knot factorization probability}

To investigate whether and to what extent composite knots factorize into separated prime components with increasing ring contour length $N$, we study the probability, $P_{s e p}\left(\tau_{c o m p}, N\right)$, of having all prime components of a composite knot of topology $\tau_{\text {comp }}$ separated along the chain. The results are plot in Fig. 4, and show that while the probability 


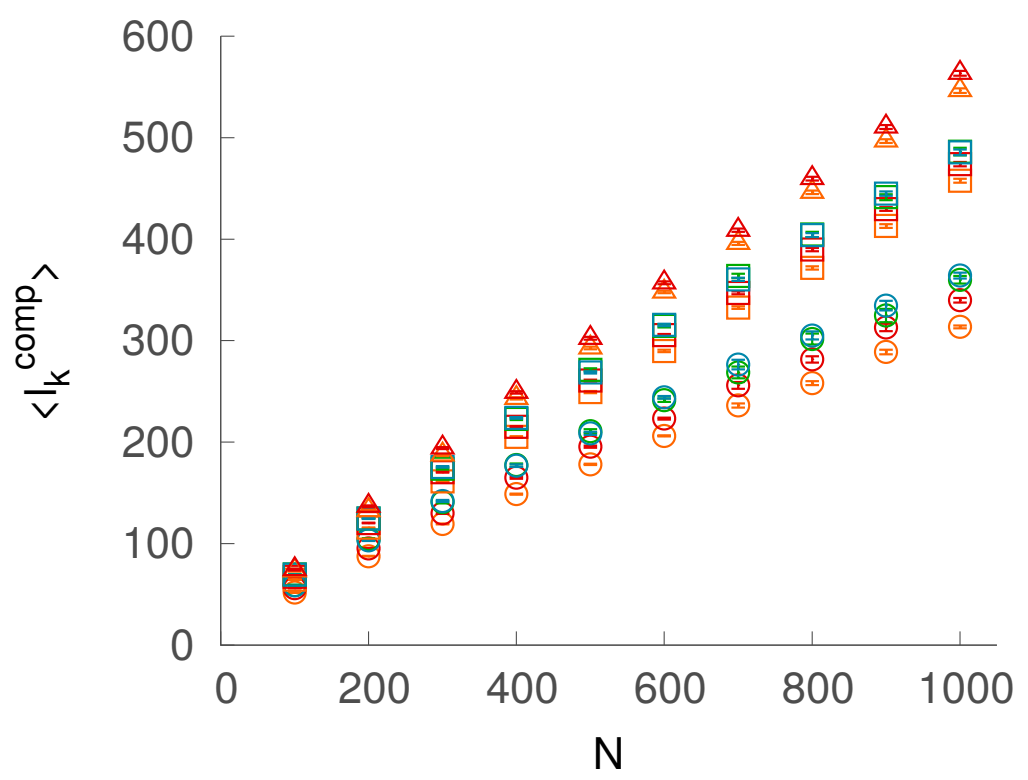

FIG. 5. From top to bottom, average lengths, $\left\langle l_{k}^{\text {comp }}\right\rangle$, of composite knots with 4,3 and 2 components. Colors and symbols follow the scheme introduced in Fig. 4.

of having all factor knots separated increases with $N$, a complete factorization is not reached in the range of lengths investigated. Indeed, even for the simplest topology, $3_{1} 3_{1}$, and the longest length, $N=1000$, which is about 100 times the typical length of a trefoil knot (see Supplementary material), the probability of having at least one non-separated component, $1-P_{\text {sep }}\left(\tau_{c o m p}, N\right)$, is still $\simeq 10 \%$. This is arguably due to the fact that knot length distributions of prime knots, although being peaked at low values of $l_{k}$ independently of $N[27,34]$ ( $l_{k} \simeq 10$ for $3_{1}$ knots, see supplementary material), have very long tails which give origin to the sublinear growth of the average knot length with $N$ (weak knot localization) [33]. Furthermore, observing the slopes of the $P_{s e p}\left(\tau_{c o m p}, N\right)$ curves, it is easily seen that not only a complete factorization is not reached in the analyzed length range, which reaches $N \gtrsim 3 N_{0}$, but it should not be expected even for far larger rings.

Comparing the results for knots with 2,3 or 4 prime components, we observe that $P_{\text {sep }}\left(\tau_{\text {comp }}, N\right)$ depends on the number of knots present and their minimum crossing number. The behavior of knots with either a $5_{1}$ or a $5_{2}$ prime component is identical, suggesting that, at least for simple prime factor knots, the only discriminant is the minimum crossing number.

\section{B. Knot lengths}

To better characterize the behavior of composite knots, we turn to study the average lengths of whole composite knots, $\left\langle l_{k}^{\text {comp }}\right\rangle$, and of their separated prime components, $\left\langle l_{k}\right\rangle$, as a function of both the contour length of the ring and the number of prime components of the knot. As reported in Fig. 5 , we observe that $\left\langle l_{k}^{\text {comp }}\right\rangle$ grows linearly in $N$, compatibly with the factor knots being randomly distributed on the ring. Fitting the lines for $N \geq 500$, we obtain the slopes reported in table I. It is clear from the reported values that the slopes depend mainly on the number of prime components forming the knot and only slightly on the exact topology of the factor knots. As a comparison, we consider the value of the knot length which would be obtained if the knots were equivalent to point-like decorations along the rings. In this limit case, $l_{k}^{c o m p}$ is given by the length of the shortest arc hosting all the decorations and depends only on the number of point-like decorations present on the ring. The expectation value of the knot length in this limit case can be computed analytically and is $\bar{l}_{2 \text { points }}=1 / 4 \mathrm{~L}, \bar{l}_{3 \text { points }}=7 / 18 \mathrm{~L}$ and $\bar{l}_{4 p o i n t s}=23 / 48 \mathrm{~L}$ for two, three and four points placed randomly on a circle of contour length $L$. The slopes obtained from our simulations are larger than those factors, showing that finite size effects are still important in the range of ring lengths considered.

Finally, looking at $\left\langle l_{k}\right\rangle$, reported in Fig. 6, we observe that the size of separated prime components is influenced by the number of prime knots tied on the ring, another consequence of the fact that knot localization is only weak. Even the average lengths of separated prime components in a $3_{1} 3_{1}$ knot is still lower than that of a prime $3_{1}$ knot, while in the asymptotic limit one would expect the lengths of prime factor knots to be independent of their number. 


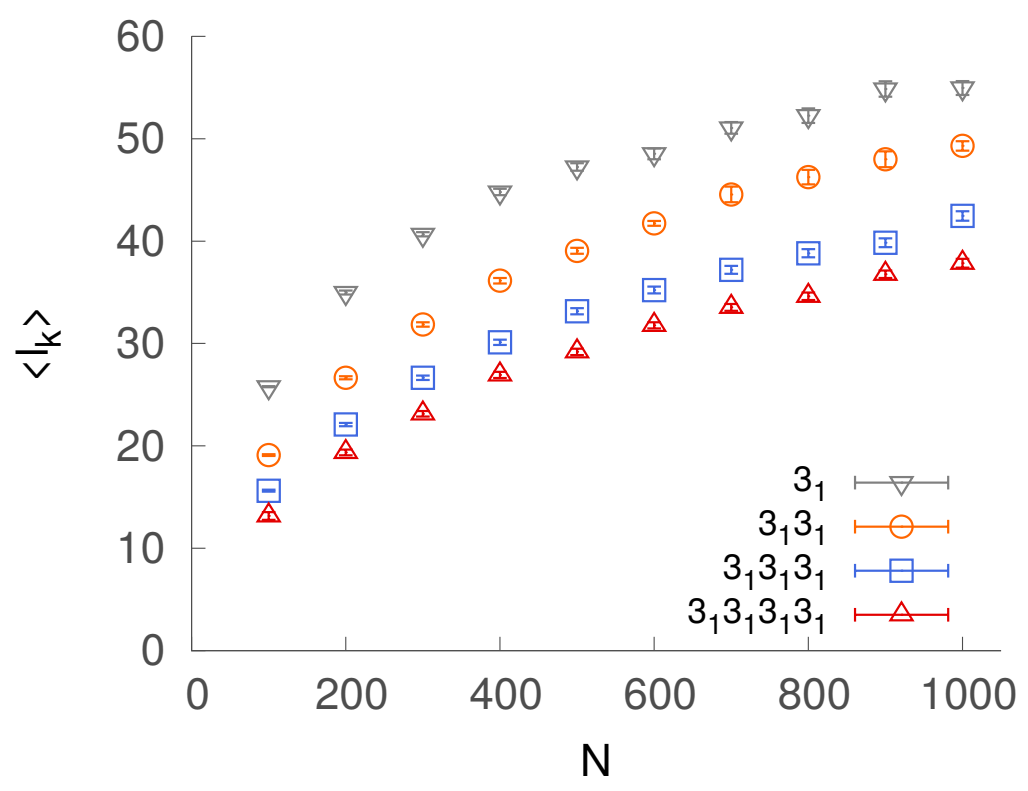

FIG. 6. From top to bottom, average knot lengths, $\left\langle l_{k}\right\rangle$, of prime trefoil knots and of trefoil factor knots in composite knots with 2, 3 and 4 components. The average is computed only on separated components.

\begin{tabular}{cccc} 
Topology & \multicolumn{3}{c}{ Number of Components } \\
& 2 & 3 & 4 \\
$(\ldots) 3_{1}$ & $0.271 \pm 0.005$ & $0.414 \pm 0.003$ & $0.504 \pm 0.004$ \\
$(\ldots) 4_{1}$ & $0.290 \pm 0.004$ & $0.423 \pm 0.003$ & $0.519 \pm 0.003$ \\
$(\ldots) 5_{1}$ & $0.294 \pm 0.006$ & $0.427 \pm 0.008$ & - \\
$(\ldots) 5_{2}$ & $0.308 \pm 0.006$ & $0.433 \pm 0.006$ & -
\end{tabular}

TABLE I. Fits obtained by linear regression of the data reported in Fig. 5 , for $N \geq 500$. The correlation coefficient is $\simeq 0.999$ for all fitting lines.

\section{Factor knots placement on the ring}

The linear growth of $\left\langle l_{k}^{c o m p}\right\rangle$ with $N$ is compatible with factor knots being randomly distributed along the ring. Nonetheless, there is still the possibility that two factor knots interact in some way when they are close to each other, for example by exchanging knot length or by slightly repelling each other because of an entropic interactions of their loops. If this is the case, we expect that some effects will be visible in the distribution of factor knot lengths, $l_{k}$, at different separations of two factor knots along the chain.

To investigate whether factor knots interact, we consider only those configurations in which all factor knots are separate. Going clockwise along the ring, for every couple of adjacent factor knots we compute their separation along the ring, $s^{A B}$, from the ending point of one factor knot to the starting point of the next one, and the sum of their knot lengths $l_{k}^{A B}=l_{k}^{A}+l_{k}^{B}$. We then average all lengths $l_{k}^{A B}$ over bins of length 10 in $s^{A B}$ and over the whole set of configurations, to observe if the average knot length of two adjacent components depends on their separation.

The results are shown in Fig. 7 for rings of length $N=1000$. One can see that going from small to large separations, the length $\left\langle l_{k}^{A B}>\right.$ has a pronounced peak at first, followed by a mild decrease which becomes more pronounced when $s^{A B} \rightarrow N$.

The behavior of $\left\langle l_{k}^{A B}\right\rangle$ may be explained without accounting for an interaction between factor knots, by considering that, since the contour length $N$ is fixed, large factor knots are forced to stay closer to each other than small ones. 


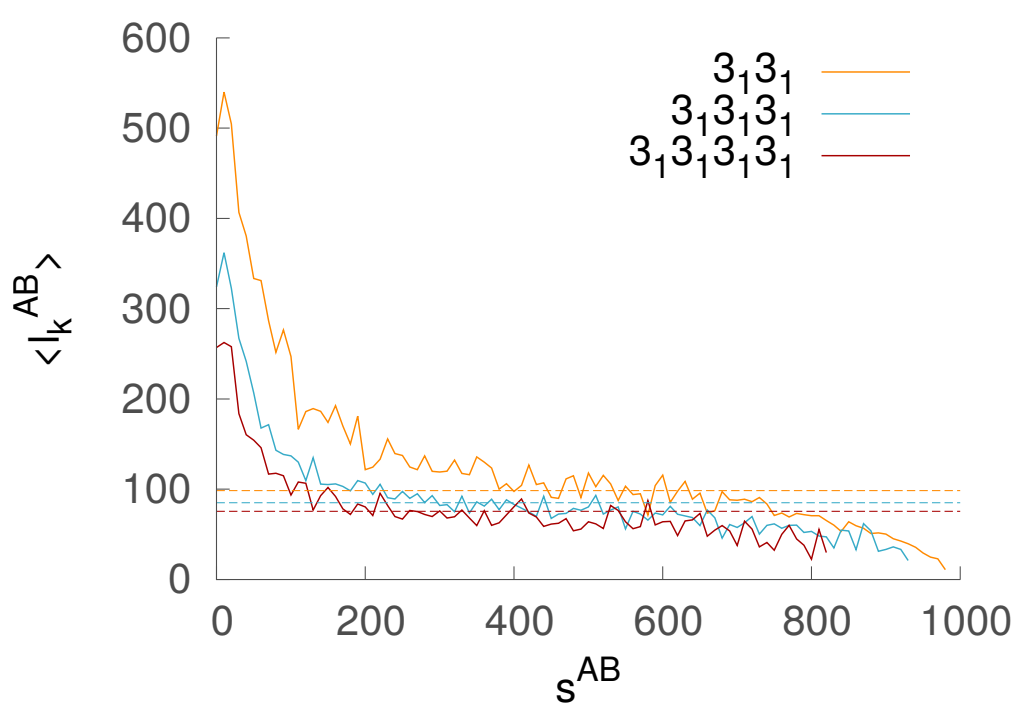

FIG. 7. Average total length of two factor knots, $\left\langle l_{k}^{A B}\right\rangle$, as a function of their separation, $s^{A B}$, along the ring, for three different composite knots on rings with $N=1000$ edges. Horizontal dashed lines correspond to the values $2 *\left\langle l_{k}\right\rangle$ reported in Fig. 6
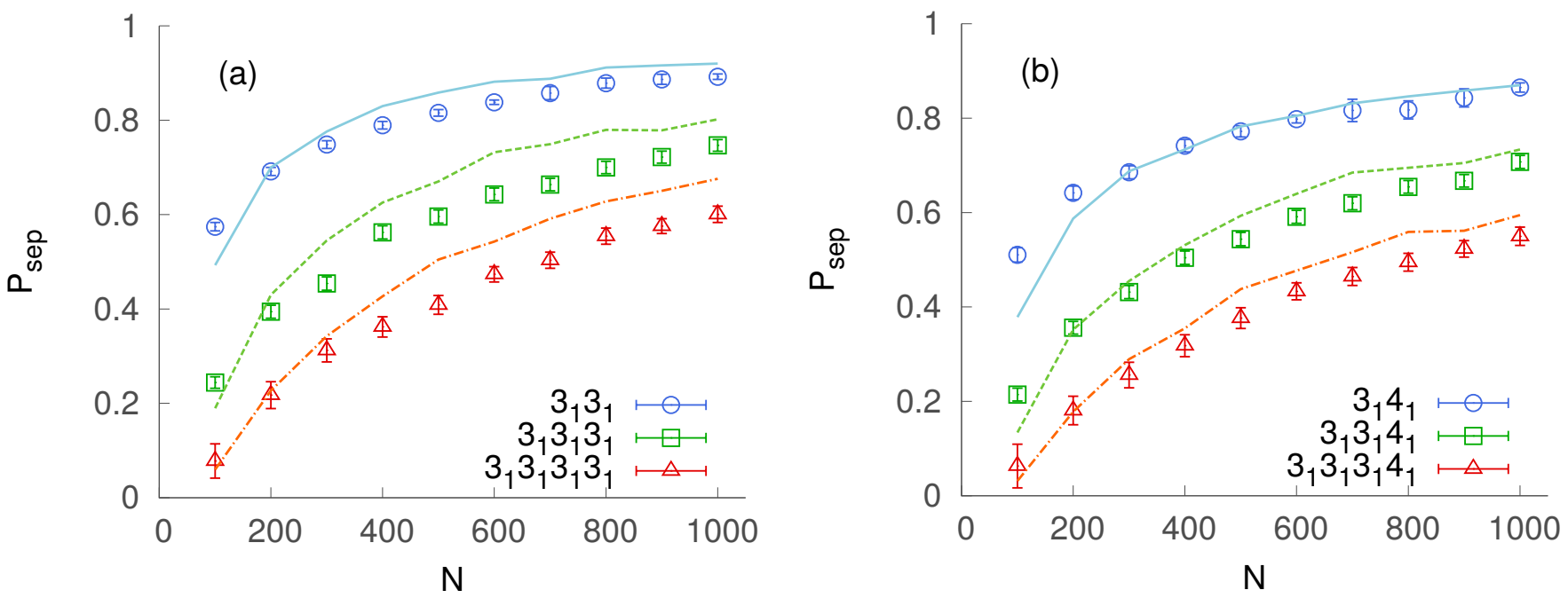

FIG. 8. Comparison between the probabilities $P_{\text {sep }}\left(\tau_{c o m p}, N\right)$ obtained from the simulations (points) and from the 1D model (lines) for composite knots made only of $3_{1}$ knots (a) and composite knots including a $4_{1}$ factor (b).

\section{DISCUSSION}

The results of Sec. III show that the paradigm of prime components behaving as decorations on the ring does not hold for FJRs in the range of length considered; nonetheless prime knots appear to be distributed randomly on the ring. Those results can be interpreted in the light of a transparent one-dimensional model, based on the following assumptions.

1. All factor knots are placed randomly on the ring.

2. Two factor knots can only be either separated along the chain or nested, see Fig. 3 b), c). This assumption lies on the observation that entangled configurations like the one depicted in Fig. 3 a),d) are very rare (less than $1 \%$ for two components knots and $N \geq 400$ ) and can, to a first approximation, be neglected.

3. The probability that a factor knot $\tau_{i}$ has length $l_{k}$ depends on the presence of other knots only through the imposition of the previous condition. In sufficiently long rings, the length distribution of factor knots can be considered to be practically identical to that of their corresponding prime knots. 

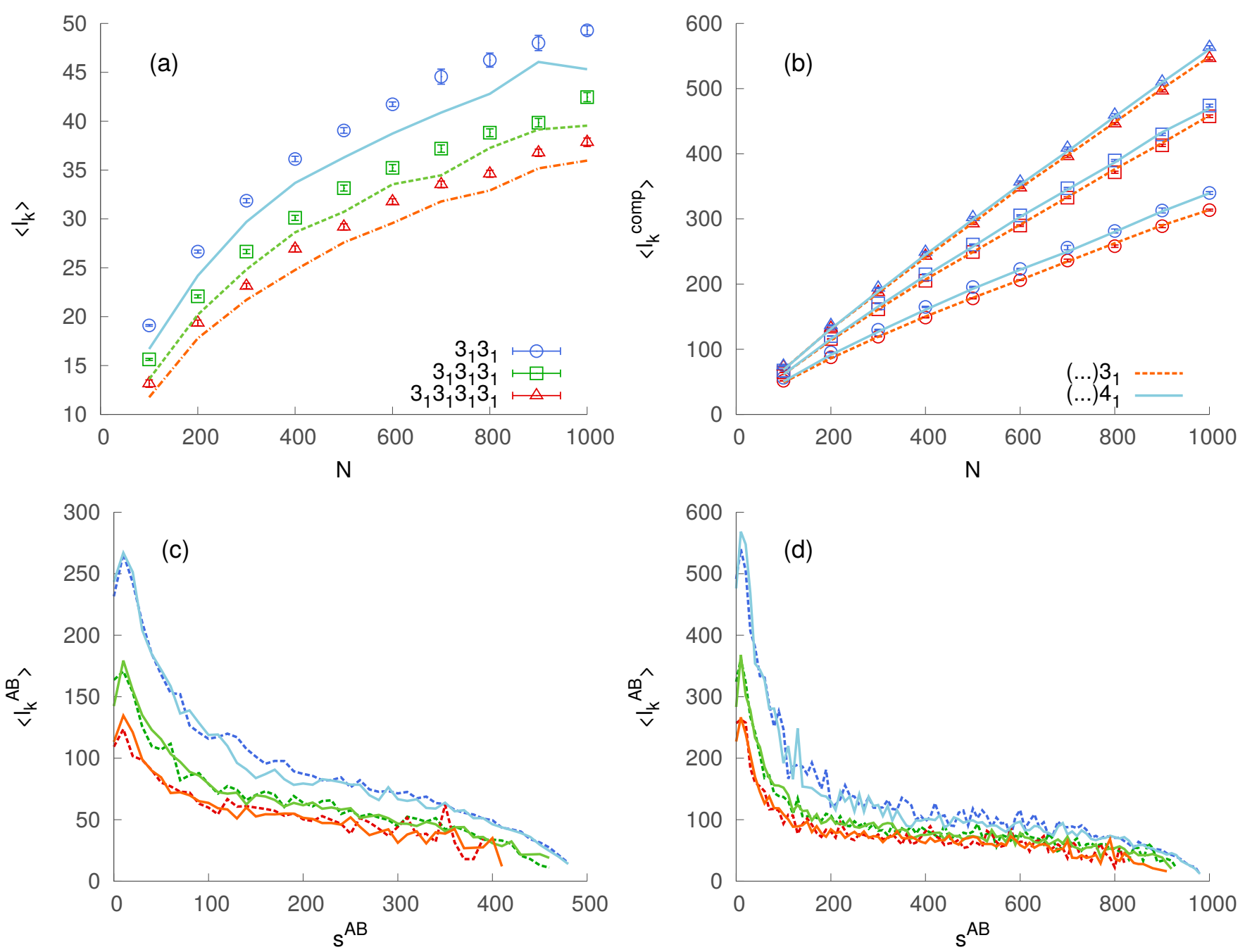

FIG. 9. (a) Length of factor knots $l_{k}$. (b) Total length of composite knots, $\left\langle l_{k}^{\text {comp }}\right\rangle$. Comparison between simulation and $1 \mathrm{D}$ model for the distribution of total knot length $\left\langle l_{k}^{A B}\right\rangle$ of couples of adjacent factor knots separate by a distance $s^{A B}$ along the ring, for rings of length $N=500$ (c) and $N=1000$ (d). Green, blue and red dashed lines report simulation data for $3_{1} 3_{1}$, $3_{1} 3_{1} 3_{1}, 3_{1} 3_{1} 3_{1} 3_{1}$ topologies. Light green, cyan and orange lines report data for the same topologies obtained from the model.

According to the first and second assumptions, we describe factor knots as paraknots placed randomly on a ring. Each paraknot is identified by a sliplink placed on the ring, joining two distant vertices of it. Every sliplink identifies a loop. No sliplink can be placed so to join a point inside a paraknot with one outside it: configurations like that depicted in Fig. $3 \mathrm{~g}$ ), j) are forbidden. According to our third hypothesis, we impose the length distribution of the paraknots to be the same as that of the prime knots they model.

Following the procedure reported in $\S I I C$, we repeated the analyses of Sec. III for the one-dimensional model. A direct comparison of the various quantities obtained from the one-dimensional model with those obtained on FJRs is reported in Fig. 8 and Fig. 9. Results from the one-dimensional model are in qualitative agreement with those obtained from the simulations for the probability of having all prime components separated, $P_{\text {sep }}\left(\tau_{c o m p}, N\right)$, reported in Fig. 8 a), b), and for the behavior of $\left\langle l_{k}\right\rangle$ with $N$ and $m$, reported in Fig. 9 a).

The agreement between the one-dimensional model and the data from simulations becomes quantitative when we take into account more global properties, like the length of the composite knot as a whole, $\left\langle l_{k}^{c o m p}\right\rangle$, and the distribution of $\left\langle l_{k}^{A B}\right\rangle$ as a function of the linear separation between adjacent couples of factor knots, $s_{A B}$, reported in Fig. 9 b), c) and $\mathrm{d})$.

The discrepancies observed between the one-dimensional model and the simulations data can be ascribed to the simplification procedure adopted to measure knot lengths in the simulations. The simplification procedure results usually in a slight overestimation of the lengths of knots. Consequently, two factor knots which are very close to each other along the chain may be seen as not being separate, lowering $P_{\text {sep }}$. Indeed, while in the case of $P_{\text {sep }}$ the 
quantities obtained from the model are systematically higher than those obtained from simulations, in the case of the length of factor knots they are systematically lower. The underestimation is expected to increase for increasing number of components, as observed in Fig. 8.

It is interesting to compare our results against previous results on the factorization of knotting probability obtained in ref. [35] for Gaussian random polygons and in ref. [37] for self-avoiding rings on the cubic lattice. In both cases the factorization was shown to hold for lengths in the order of $\simeq N_{0}$. The results reported in Sec. III, in which no complete factorization into separated prime components was observed, and their agreement with the model presented in this section, raise the question if the "decorated ring" assumption [35-37] is really necessary to guarantee the factorization of the knotting probability of composite knots into the product of those of their prime components, or if a mechanism like the one introduced by our one-dimensional model will be sufficient.

It is also interesting to consider what happens when one studies an equilibrium population of composite knots obtained from ring polymers circularized at $\theta$-point. Our results show that the probability $P_{\text {sep }}\left(\tau_{\text {comp }}, N\right)$ decreases with the number of prime components tied on a ring of length $N$. Since for increasing ring length the average number of prime components involved in a composite knot increases (see Fig. 2), it is possible that in such sample no complete factorization into separated prime components will be observed, on average, at any ring contour length.

\section{SUMMARY AND CONCLUSIONS}

We have presented a numerical study of the properties of composite knots made of up to 4 components. Using advanced knot localization methods, we have been able to identify separated prime components, measure their length and separation, as well as the probability of having a full factorization of composite knots into separated prime components.

We have shown that a complete factorization of a composite knot into separate prime components is not to be expected in the range of lengths considered; nonetheless the characteristics of composite knots are compatible with a random placement of their prime components along the ring. We rationalized the simulation results using a simple onedimensional model, in which factor knots are substituted with randomly placed paraknots whose length distribution coincide with that of the factor knots.

Interestingly, the range of ring contour lengths considered in this study is comparable, in relation with the unknotting length, to those considered in previous studies [35, 37], in which the factorization of the knotting probability of composite knots was observed in two different polymer systems: Gaussian polygons [35] ( no excluded volume) and self-avoiding rings on the square lattice [37]. Following this observation we suggest that, at least for polymer models without excluded volume, the "decorated ring" paradigm is not necessary to explain the factorization of knot probabilities, and may be substituted by the "paraknot" paradigm used to explain our data. Since our study was performed on infinitely thin polymer rings, it will be interesting to investigate whether the introduction of excluded volume leads to a faster knot factorization, and thus to the "decorated ring" paradigm, or if the behavior in the presence of excluded volume is still compatible with the one reported here.

Finally, it is known that geometrical confinement affects knot properties, including knot localization [21, 47, 48]. In particular, prime knots confined into nano-channels [21] and quasi two dimensional knots [29, 30] are much more localized that their unconstrained counterparts studied here. An higher degree of localization intuitively leads to a more effective factorization of composite knots into separated prime components and, in fact, in such cases, composite knots have been shown to be completely factorized, and therefore equivalent to decorated rings [21, 30]. We think it will be interesting to extend the present work to study the progressive onset of knot factorization as a function of increasing geometrical confinement.

\section{ACKNOWLEDGEMENTS}

I am deeply indebted to C. Micheletti for his support in this project. I am also very grateful to E. Orlandini and E. Paoli for several useful discussions. I acknowledge financial support from the Slovene Agency for Research and Development ( Grant No. J1-4134).

[1] J. des Cloizeaux, J. Phys. Lett. 42, L433 (1981).

[2] N. T. Moore, R. C. Lua, and A. Y. Grosberg, Proceedings of the National Academy of Sciences of the United States of America 101, 13431 (2004). 
[3] A. Stasiak, V. Katritch, J. Bednar, D. Michoud, and J. Dubochet, Nature 384, 122 (1996).

[4] C. Weber, A. Stasiak, P. D. L. Rios, and G. Dietler, Biophys. J. 90, 3100 (2006).

[5] E. Orlandini, A. L. Stella, and C. Vanderzande, Phys Rev E Stat Nonlin Soft Matter Phys 82, 050804 (2010).

[6] A. M. Saitta, P. D. Soper, E. Wasserman, and M. L. Klein, Nature 399, 46 (1999).

[7] Y. Arai, R. Yasuda, K. Akashi, Y. Harada, H. Miyata, T. Kinosita, and H. Itoh, Nature 399, 446 (1999).

[8] A. Bates and A. Maxwell, DNA Topology (Oxford Bioscience, 2005).

[9] D. Meluzzi, D. Smith, and G. Arya, Annual review of biophysics 39, 349 (2010).

[10] W. Taylor, Nature 406, 916 (2000).

[11] P. Virnau, L. A. Mirny, and M. Kardar, PLoS Comput Biol 2, e122 (2006).

[12] N. P. King, E. O. Yeates, and T. O. Yeates, Journal of Molecular Biology 373, 153 (2007).

[13] R. Potestio, C. Micheletti, and H. Orland, PLoS computational biology 6, e1000864 (2010).

[14] T. Škrbić, C. Micheletti, and P. Faccioli, PLoS computational biology 8, e1002504 (2012).

[15] S. a Beccara, T. Škrbić, R. Covino, C. Micheletti, and P. Faccioli, PLoS computational biology 9, e1003002 (2013).

[16] J. Arsuaga, M. Vzquez, S. Trigueros, D. W. Sumners, and J. Roca, Proceedings of the National Academy of Sciences 99, 5373 (2002), http://www.pnas.org/content/99/8/5373.full.pdf+html.

[17] J. Arsuaga, M. Vazquez, P. McGuirk, S. Trigueros, D. W. Sumners, and J. Roca, Proceedings of the National Academy of Sciences of the United States of America 102, 9165 (2005), http://www.pnas.org/content/102/26/9165.full.pdf+html.

[18] D. Marenduzzo, E. Orlandini, A. Stasiak, D. W. Sumners, L. Tubiana, and C. Micheletti, Proceedings of the National Academy of Sciences 106, 22269 (2009), http://www.pnas.org/content/106/52/22269.full.pdf+html.

[19] D. Reith, P. Cifra, A. Stasiak, and P. Virnau, Nucleic acids research 40, 5129 (2012).

[20] C. Micheletti, D. Marenduzzo, and E. Orlandini, Physics Reports 504, 1 (2011).

[21] C. Micheletti and E. Orlandini, Soft Matter 8, 10959 (2012).

[22] A. Rosa, M. Di Ventra, and C. Micheletti, Physical Review Letters 109, 118301 (2012).

[23] I. Coluzza, P. D. J. van Oostrum, B. Capone, E. Reimhult, and C. Dellago, Physical Review Letters 110, 075501 (2013).

[24] D. W. Sumners and S. G. Whittington, Journal of Physics A: Mathematical and General 21, 1689 (1988).

[25] E. Ben-Naim, Z. A. Daya, P. Vorobieff, and R. E. Ecke, Phys. Rev. Lett. 86, 1414 (2001).

[26] P. Virnau, Y. Kantor, and M. Kardar, Journal of the American Chemical Society 127, 15102 (2005), http://pubs.acs.org/doi/pdf/10.1021/ja052438a.

[27] L. Tubiana, A. Rosa, F. Fragiacomo, and C. Micheletti, Macromolecules 46, 3669 (2013), http://pubs.acs.org/doi/pdf/10.1021/ma4002963.

[28] Y. Diao, N. Pippenger, and D. W. Sumners, Journal of knot theory and its ramifications 3, 419 (1994).

[29] E. Guitter and E. Orlandini, Journal of Physics A: Mathematical and General 32, 1359 (1999).

[30] R. Metzler, A. Hanke, P. G. Dommersnes, Y. Kantor, and M. Kardar, Phys. Rev. Lett. 88, 188101 (2002).

[31] P. G. Dommersnes, Y. Kantor, and M. Kardar, Physical Review E 66, 031802 (2002).

[32] V. Katritch, W. K. Olson, A. Vologodskii, J. Dubochet, and A. Stasiak, Phys. Rev. E 61, 5545 (2000).

[33] B. Marcone, E. Orlandini, A. L. Stella, and F. Zonta, Physical Review E 75, 041105 (2007).

[34] M. L. Mansfield and J. F. Douglas, J. Chem. Phys. 133, 044903 (2010).

[35] K. Tsurusaki and T. Deguchi, Journal of the Physical Society of Japan 64, 1506 (1995).

[36] E. Orlandini, M. C. Tesi, E. J. J. van Rensburg, and S. G. Whittington, Journal of Physics A: Mathematical and General 31, 5953 (1998).

[37] M. Baiesi, E. Orlandini, and A. L. Stella, Journal of Statistical Mechanics: Theory and Experiment 2010, P06012 (2010).

[38] L. Tubiana, E. Orlandini, and C. Micheletti, Progress of Theoretical Physics Supplement 191, 192 (2011).

[39] R. Metzler, A. Hanke, P. G. Dommersnes, Y. Kantor, and M. Kardar, Phys. Rev. E 65, 061103 (2002).

[40] S. Alvarado, J. Calvo, and K. Millett, Journal of Statistical Physics 143, 102 (2011), 10.1007/s10955-011-0164-4.

[41] M. K. Shimamura and T. Deguchi, Physics Letters A 274, 184 (2000).

[42] J. Hoste and M. Thistlethwaite, Knotscape (1999).

[43] A. S. K. Millett, A. Dobay, Macromolecules 38, 601 (2005).

[44] E. Orlandini and S. G. Whittington, Rev. Mod. Phys. 79, 611 (2007).

[45] K. Millett, Journal of Knot Theory and its Ramifications 19, 601 (2010).

[46] K. Koniaris and M. Muthukumar, Phys. Rev. Lett. 66, 2211 (1991).

[47] L. Tubiana, E. Orlandini, and C. Micheletti, Phys. Rev. Lett. 107, 188302 (2011).

[48] C. Micheletti and E. Orlandini, Macromolecules 45, 2113 (2012). 


\section{SUPPLEMENTARY INFORMATION}
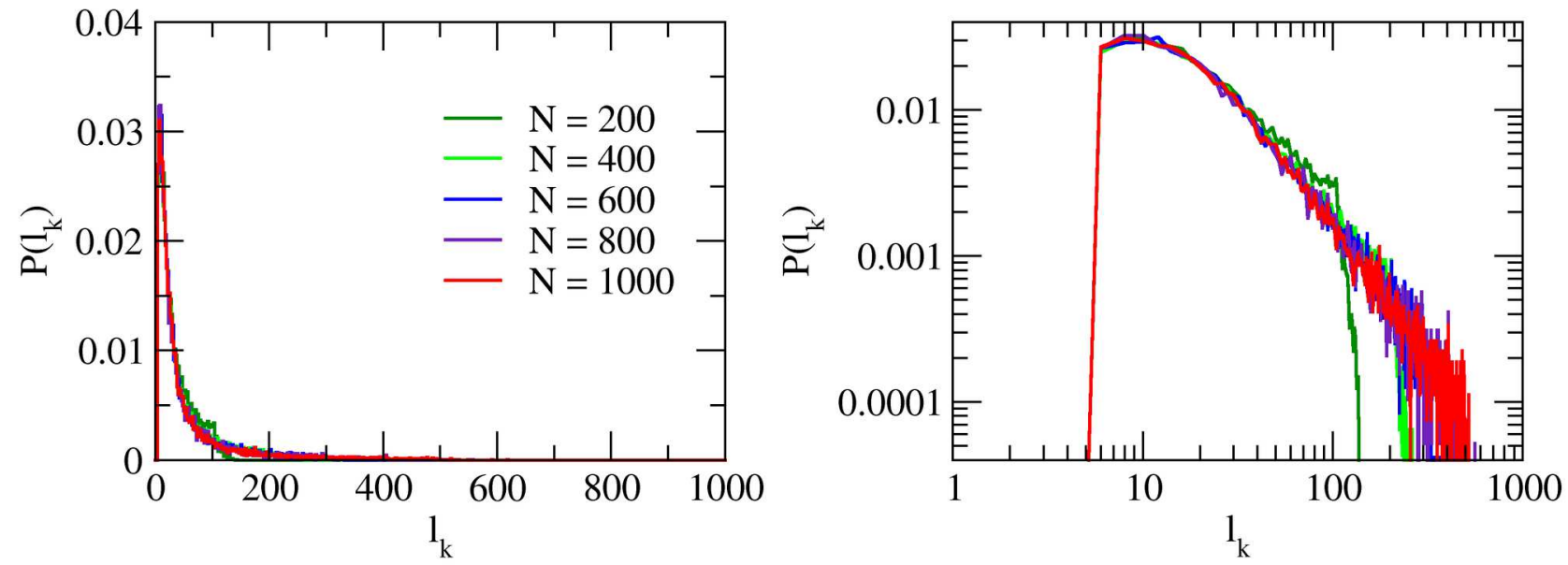

SUPPL. FIG. 1. Knot length distribution for trefoil knots tied on rings of increasing length $N$. Note that all distributions are peaked on the same value $l_{k} \simeq 10$ while they differ because of their tails. 

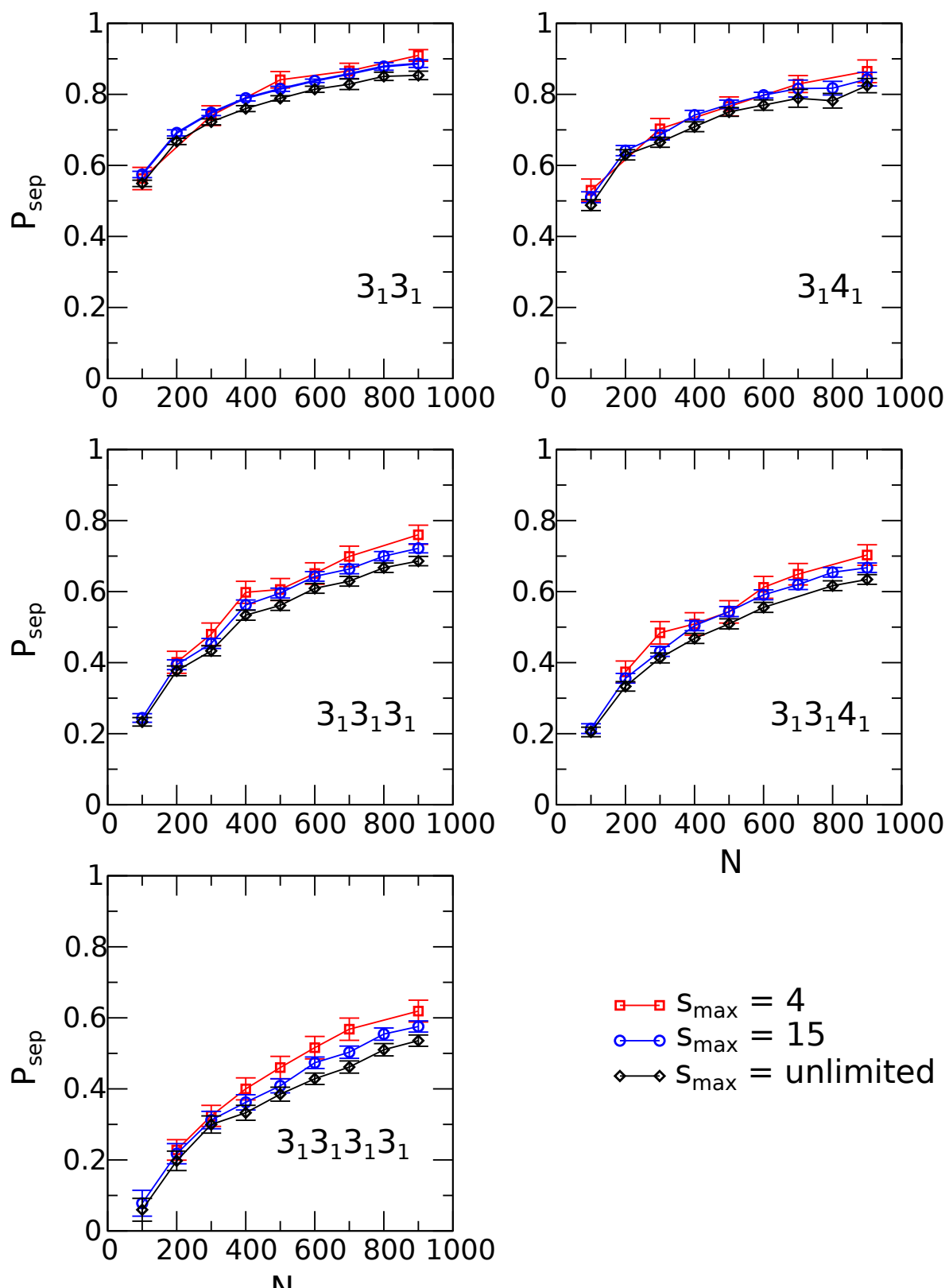

$$
\begin{aligned}
& \because S_{\max }=4 \\
& \leftrightarrow S_{\max }=15 \\
& \leftrightarrow S_{\max }=\text { unlimited }
\end{aligned}
$$

$\mathrm{N}$ 